\title{
ON INTUITIONISTIC FUZZY IDEALS BITOPOLOGICAL SPACES
}

\author{
Mohammed Jassim Tuaimah ${ }^{1}$, Mohammed Jassim Mohammed $^{2}$ \\ 1 Department of Mathematics, College of Education for Pure Science, Thi-Qar University, Thi-Qar , Iraq \\ ${ }^{2}$ Department of Mathematics, College of Education for Pure Science , Thi-Qar University, Thi-Qar , Iraq
}

\section{ABSTRACT}

In this paper we introduce the notion of intuitionistic fuzzy ideals in intuitionistic fuzzy bitopological spaces and we prove some results about it.

\section{Keywords}

Intuitionistic fuzzy bitopological space; intuitionistic fuzzy ideal and intuitionistic fuzzy local function .

\section{Council for Innovative Research}

Peer Review Research Publishing System

Journal: JOURNAL OF ADVANCES IN MATHEMATICS

Vol.11, No.6

editorjam@gmail.com , www.cirjam.com 


\section{INTRODUCTION :}

The concept of "fuzzy sets" was first introduced by Zadeh [7] in 1965. Atanassov [1] in 1983, initiated the study of an "intuitionistic fuzzy sets" and defined the "interior and closure "over the set of intuitionistic fuzzy sets. In 1995, Coker and Demirci [3], constructed the basic concept so called "intuitionistic fuzzy points " and related objects as " quasi-coincidence ". Kandil , Nouh and El-Sheikh [5] in 1995, initiated the study of " fuzzybitopological spaces ". In 1997 , Coker [4] defined the concept of " intuitionistic fuzzy topology ". The concept of " fuzzy ideals in fuzzy bitopological space and fuzzy pairwise local function" were first introduced by AbdEl_Monsef , Kozae , Salama and Elagmy [2] in 2012. The notions of " intuitionistic fuzzy ideals and intuitionistic fuzzy local functions "studied by Salama and Alblowi [6] in 2012 .

In this paper we introduce the notion of an "intuitionistic fuzzy bitopological spaces " and study the concept of an "intuitionistic fuzzy ideals, intuitionistic fuzzy local functions in intuitionistic fuzzy ideal bitopological spaces" and prove some results about them.

\section{PRELIMINARIES :}

\section{Definition. 2.1. [7] :}

Let $X$ be a non-empty set and $I=[0,1]$ be the closed interval of the real numbers. A fuzzy subset $\mu$ of $X$ is defined to be a membership function $\mu: X \rightarrow I$, such that $\mu(x) \in I$ for every $x \in X$. The set of all fuzzy subsets of $X$ denoted by $I^{X}$.

\section{Definition. 2.2. [1] :}

An intuitionistic fuzzy set (IFS, for short) $A$ is an object have the form : $A=\left\{\left\langle x, \mu_{A}(x), v_{A}(x)\right\rangle ; x \in X\right\}$, where the functions $\mu_{A}: X \rightarrow I, v_{A}: X \rightarrow I$ denote the degree of membership and the degree of non-membership of each element $x \in X$ to the set $A$ respectively, and $0 \leq \mu_{A}(x)+v_{A}(x) \leq 1$, for each $x \in X$. The set of all intuitionistic fuzzy sets in $X$ denoted by $\operatorname{IFS}(X)$.

\section{Definition. 2.3. [4] :}

$0_{\sim}=\langle x, 0,1\rangle, 1_{\sim}=\langle x, 1,0\rangle$ are the intuitionistic fuzzy sets corresponding to empty set and the entire universe respectively

\section{Definition. 2.4. [3] :}

Let $X$ be a non-empty set. An intuitionistic fuzzy point (IFP, for short) denoted by $x_{(\alpha, \beta)}$ is an intuitionistic fuzzy set have the form : $x_{(\alpha, \beta)}(y)=\left\{\begin{array}{ll}\langle x, \alpha, \beta\rangle & ; x=y \\ \langle x, 0,1\rangle & ; x \neq y\end{array}\right.$, where $x \in X$ is a fixed point, and $\alpha, \beta \in[0,1]$ satisfy $\alpha+\beta \leq 1$. The set of all IFPs denoted by $\operatorname{IFP}(X)$. If $A \in \operatorname{IFS}(X)$, we say that $x_{(\alpha, \beta)} \in A$ if and only if $\alpha \leq \mu_{A}(x)$ and $\beta \geq v_{A}(x)$, for each $x \in X$.

\section{Definition. 2.5. [3] :}

Let $A=\left\langle x, \mu_{A}(x), v_{A}(x)\right\rangle, B=\left\langle x, \mu_{B}(x), v_{B}(x)\right\rangle$ be two IFSs in $X$ and $x_{(\alpha, \beta)} \in \operatorname{IFP}(X)$, then :

(1) $A$ is said to be quasi-coincident with $B$ (written $A q B$ ) if there exists an element $x \in X$, such that $\mu_{A}(x)+$ $\mu_{B}(x)>1$ and $v_{A}(x)+v_{B}(x)<1$. Otherwise $A$ is not quasi-coincident with $B$ and denoted by $A \bar{q} B$.

(2) We say that $A$ is quasi- coincident with $x_{(\alpha, \beta)}$, denoted by $x_{(\alpha, \beta)} q A$ if $\mu_{A}(x)+\alpha>1$ and $v_{A}(x)+\beta<1$.

\section{Definition.2.6. [4] :}

An intuitionistic fuzzy topology (IFT, for short) on a non-empty set $X$ is a family $\tau$ of an intuitionistic fuzzy sets in $X$ such that: 

(i) $0_{\sim}, 1_{\sim} \in \tau$,
(ii) $G_{1} \cap G_{2} \in \tau$, for any $G_{1}, G_{2} \in \tau$,
(iii) $\cup G_{i} \in \tau$, for any arbitrary family $\left\{G_{i}: i \in \Gamma\right\} \subseteq \tau$.

In this case the pair $(\mathrm{X}, \tau)$ is called an intuitionistic fuzzy topological space (IFTS, in short) .

\section{Definition. 2.7. [6] :}

A non-empty collection of intuitionistic fuzzy sets $L$ of a set $X$ is called intuitionistic fuzzy ideal on $X$ ( IFI, for short) such that :

(i) If $A \in L$ and $B \leq A \Rightarrow B \in L$ ( heredity),

(ii) If $A \in L$ and $B \in L \Rightarrow A \vee B \in L$ ( finite additivity).

If $(X, \tau)$ be an IFTS, then the triple $(X, \tau, L)$ is called an intuitionistic fuzzy ideal topological space (IFITS, for short ) .

\section{Definition. 2.8. [6] :}

Let $(X, \tau, L)$ be an IFITS. If $A \in \operatorname{IFS}(X)$, then the intuitionistic fuzzy local function $A^{\star}(L, \tau)\left(A^{\star}\right.$, for short) of $A$ in $(X, \tau, L)$ is the union of all intuitionistic fuzzy points $x_{(\alpha, \beta)}$ such that :

$A^{\star}(L, \tau)=\vee\left\{x_{(\alpha, \beta)}: A \wedge U \notin L\right.$, for every $\left.U \in N\left(x_{(\alpha, \beta)}, \tau\right)\right\}$, where $N\left(x_{(\alpha, \beta)}, \tau\right)$ is the set of all quasi-neighborhoods of an $I F P x_{(\alpha, \beta)}$ in $\tau$. The intuitionistic fuzzy closure operator of an IFS $A$ is defined by $c l^{\star}(A)=A \vee A^{\star}$, and $\tau^{\star}(L)$ is an IFT finer than $\tau$ generated by $c l^{\star}($.$) and defined as \tau^{\star}(L)=\left\{A: c l^{\star}\left(A^{c}\right)=A^{c}\right\}$.

\section{Definition. 2.9. [5] :}

A fuzzy bitopological space $\left(X, \mathcal{T}_{1}, \mathcal{T}_{2}\right)(F B T S$, for short $)$ is a non-empty set $X$ equipped with two fuzzy topologies $\mathcal{T}_{1}$ and $\mathcal{T}_{2}$.

\section{Definition. 2.10. [2] :}

A fuzzy set $\mu$ in a $\operatorname{FBTS}\left(X, \mathcal{T}_{1}, \mathcal{T}_{2}\right)$ is called pairwise quasi-neighborhood of a fuzzy point $x_{t}$ if and only if there exists a $\mathcal{T}_{\mathrm{i}}$-fuzzy open set $v, \mathrm{i} \in\{1,2\}$ such that $x_{t} q v \leq \mu$. The set of all pairwise quasi-neighborhoods of $x_{t}$ in $\mathcal{T}_{\mathrm{i}}$ denoted by $P N\left(x_{t}, \mathcal{T}_{i}\right)$.

\section{Definition. 2.11. [2] :}

Let $\left(X, \mathcal{T}_{1}, \mathcal{T}_{2}\right)$ be a FBTS and $\mathcal{L}$ be a fuzzy ideal on $X$, then $\left(X, \mathcal{T}_{1}, \mathcal{T}_{2}, \mathcal{L}\right)$ is called fuzzy ideal bitopological space (FIBTS ). The pairwise fuzzy local function $P \mu^{\star}\left(\mathcal{L}, \mathcal{T}_{i}\right)$ of a fuzzy set $\mu$ is the union of all fuzzy points $x_{t}$ such that if $\rho \in P N\left(x_{t}, \mathcal{T}_{i}\right), i \in\{1,2\}$ and $\gamma \in \mathcal{L}$, then there is at least one $r \in X$ for which $\rho(r)+\mu(r)-1>\gamma(r)$. The pairwise fuzzy closure operator of a fuzzy set $\mu$ is defined by $\mathcal{T}_{i}-c l^{\star}(\mu)=\mu \vee P \mu^{\star}\left(\mathcal{L}, \mathcal{T}_{i}\right)$ and $\mathcal{T}_{i}^{\star}(\mathcal{L})$ is the fuzzy bitopology finer than $\mathcal{T}_{i}$ generated by $\mathcal{T}_{i}-c l^{\star}($.$) and defined as follows : \mathcal{T}_{i}^{\star}(\mathcal{L})=\left\{\mu: \mathcal{T}_{i}-c l^{\star}\left(\mu^{c}\right)=\mu^{c}\right\}$.

\section{SOME RESULTS OF INTUITIONISTIC FUZZY IDEALS BITOPOLOGICAL SPACES}

\section{Definition. 3.1 :}

Let $\tau_{1}$ and $\tau_{2}$ be two intuitionistic fuzzy topologies on a non-empty set $X$. The triple $\left(X, \tau_{1}, \tau_{2}\right)$ is called an "intuitionistic fuzzy bitopological space" (IFBTS, for short), every member of $\tau_{i}$ is called $\tau_{i}$-intuitionistic fuzzy open set $\left(\tau_{i}-\right.$ IFOS $), i \in\{1,2\}$ and the complement of $\tau_{i}$-IFOS is $\tau_{i}$-intuitionistic fuzzy closed set $\left(\tau_{i}-I F C S\right)$, $i \in\{1,2\}$. 


\section{Example. 3.2 :}

Let $X=\{e, d\}$ and $A, B \in I F S(X)$ such that : $A=\langle x,(0.3,0.1),(0.5,0.6)\rangle, B=\langle x,(0.2,0.4),(0.7,0.3)\rangle$.

Let $\tau_{1}=\left\{0_{\sim}, 1_{\sim}, A\right\}$ and $\tau_{2}=\left\{0_{\sim}, 1_{\sim}, B\right\}$ be two IFTs on $X$. Then $\left(X, \tau_{1}, \tau_{2}\right)$ is IFBTS.

\section{Definition. 3.3 :}

Let $\left(X, \tau_{1}, \tau_{2}\right)$ be an IFBTS $A \in \operatorname{IFS}(X)$ and $x_{(\alpha, \beta)} \in \operatorname{IFP}(X)$. Then $A$ is said to be quasi-neighborhood of $x_{(\alpha, \beta)}$ if there exists a $\tau_{i}-\operatorname{IFOS} B, i \in\{1,2\}$ such that $x_{(\alpha, \beta)} q B \leq A$. The set of all quasi-neighborhoods of $x_{(\alpha, \beta)}$ in $\left(X, \tau_{1}, \tau_{2}\right)$ is denoted by $N\left(x_{(\alpha, \beta)}, \tau_{i}\right), i \in\{1,2\}$.

\section{Definition. 3.4 :}

An intuitionistic fuzzy bitopological space $\left(X, \tau_{1}, \tau_{2}\right)$ with an intuitionistic fuzzy ideal $L$ on $X$ is called " intuitionistic fuzzy ideal bitopological space" $\left(X, \tau_{1}, \tau_{2}, L\right)$ and denoted by IFIBTS .

\section{Example. 3.5:}

Let $X=\{e\}$ and $A, B \in \operatorname{IFS}(X)$ such that : $A=\langle x, 0.3,0.5\rangle, B=\langle x, 0.2,0.4\rangle$. Let $\left(X, \tau_{1}, \tau_{2}\right)$ be an IFBTS, where $\tau_{1}=\left\{0_{\sim}, 1_{\sim}, A\right\}$ and $\tau_{2}=\left\{0_{\sim}, 1_{\sim}, B\right\}$. If $L=\left\{0_{\sim}, A, C: C \in \operatorname{IFS}(X)\right.$ and $\left.C \leq A\right\}$ be an IFI on $X$. Then $\left(X, \tau_{1}, \tau_{2}, L\right)$ is IFIBTS.

\section{Definition. 3.6 :}

Let $\left(X, \tau_{1}, \tau_{2}, L\right)$ be an IFIBTS and $A \in \operatorname{IFS}(X)$. Then the " intuitionistic fuzzy local function " of $A$ in $\left(X, \tau_{1}, \tau_{2}, L\right)$ denoted by $A^{\star}\left(L, \tau_{i}\right), i \in\{1,2\}$ and defined as follows : $A^{\star}\left(L, \tau_{i}\right)=\vee\left\{x_{(\alpha, \beta)}: A \wedge U \notin L\right.$, for every $\left.U \in N\left(x_{(\alpha, \beta)}, \tau_{i}\right)\right\}$.

\section{Definition. 3.7 :}

An " intuitionistic fuzzy local function " $A^{\star}(L, \delta)$ having the form : $A^{\star}(L, \delta)=\vee\left\{x_{(\alpha, \beta)}: A \wedge U \notin L\right.$, for every $U \in$ $\left.N\left(x_{(\alpha, \beta)}, \delta\right)\right\}$, where $\delta=\tau_{1} \vee \tau_{2}$ is an intuitionistic fuzzy topology generated by $\tau_{1}, \tau_{2}$ and $N\left(x_{(\alpha, \beta)}, \delta\right)$ is the set of all quasi-neighborhoods of $x_{(\alpha, \beta)}$ in $\delta$.

\section{Definition. 3.8 :}

Let $\left(X, \tau_{1}, \tau_{2}\right)$ be an IFBTS and $A \in \operatorname{IFS}(X)$. Then " intuitionistic fuzzy interior " and "intuitionistic fuzzy closure " of $A$ with respect to $\tau_{i}, i \in\{1,2\}$ are defined by : $\tau_{i}-\operatorname{int}(A)=\mathrm{V}\left\{G: G\right.$ is $\left.a \tau_{i}-I F O S, G \leq A\right\}$, $\tau_{i}-\operatorname{cl}(A)=\Lambda\left\{K: K\right.$ is a $\left.\tau_{i}-I F C S, A \leq K\right\}$

\section{Remark. 3.9 :}

We denote $\delta-\operatorname{int}(A), \delta-\operatorname{cl}(A)$ the interior and closure of $A$ respectively, with respect to $\delta=\tau_{1} \vee \tau_{2}$.

\section{Proposition. 3.10 :}

Let $\left(X, \tau_{1}, \tau_{2}\right)$ be an IFBTS and $A \in \operatorname{IFS}(X)$. Then we have :

(i) $\tau_{i}-\operatorname{int}(A) \leq A, i \in\{1,2\}$.

(ii) $\tau_{i}-\operatorname{int}(A)$ is a largest $\tau_{i}-I F O S$ contains in $A$.

(iii) $A$ is a $\tau_{i}-$ IFOS if and only if $\tau_{i}-\operatorname{int}(A)=A$.

(iv) $\tau_{i}-\operatorname{int}\left(\tau_{i}-\operatorname{int}(A)\right)=\tau_{i}-\operatorname{int}(A)$. 
(v) $A \leq \tau_{i}-\operatorname{cl}(A), i \in\{1,2\}$.

(vi) $\tau_{i}-\operatorname{cl}(A)$ is a smallest $\tau_{i}-I F C S$ contains $A$.

(vii) $A$ is a $\tau_{i}-I F C S$ if and only if $\tau_{i}-\operatorname{cl}(A)=A$.

(viii) $\tau_{i}-\operatorname{cl}\left(\tau_{i}-\operatorname{cl}(A)\right)=\tau_{i}-\operatorname{cl}(A)$.

(ix) $\left[\tau_{i}-\operatorname{int}(A)\right]^{c}=\tau_{i}-\operatorname{cl}\left(A^{c}\right), i \in\{1,2\}$.

(x) $\left[\tau_{i}-\operatorname{cl}(A)\right]^{c}=\tau_{i}-\operatorname{int}\left(A^{c}\right), i \in\{1,2\}$.

Proof : Clearly .

\section{Definition. 3.11 :}

We define " $\star$-intuitionistic fuzzy closure operator " for intuitionistic fuzzy bitopology $\tau_{i}^{\star}(L)$ as follows : $\tau_{i}-$ $c l^{\star}(A)=A \vee A^{\star}\left(L, \tau_{i}\right)$ for every $A \in \operatorname{IFS}(X)$. Also, $\tau_{i}^{*}(L)$ is called an intuitionistic fuzzy bitopology generated by $\tau_{i}-c l^{\star}(A)$ and defined as : $\tau_{i}^{\star}(L)=\left\{A: \tau_{i}-c l^{\star}\left(A^{c}\right)=A^{c}, i \in\{1,2\}\right\}$.

Note : $\tau_{i}^{\star}(L)$ finer than intuitionistic fuzzy bitopology $\tau_{i}$, (i.e. $\left.\tau_{i} \leq \tau_{i}^{\star}(L)\right)$.

\section{Remark. 3.12 :}

(i) If $L=\left\{0_{\sim}\right\} \Rightarrow A^{\star}\left(L, \tau_{i}\right)=\tau_{i}-\operatorname{cl}(A)$, for any $A \in \operatorname{IFS}(X)$

$\Rightarrow \tau_{i}-c l^{\star}(A)=A \vee A^{\star}\left(L, \tau_{i}\right)=A \vee \tau_{i}-\operatorname{cl}(A)=\tau_{i}-c l(A) \Rightarrow \tau_{i}^{\star}\left(\left\{0_{\sim}\right\}\right)=\tau_{i}, i \in\{1,2\}$.

(ii) If $L=I F S(X) \Rightarrow A^{\star}\left(L, \tau_{i}\right)=0_{\sim}$, for any $A \in \operatorname{IFS}(X) \Rightarrow \tau_{i}-c l^{\star}(A)=A \vee A^{\star}\left(L, \tau_{i}\right)=A \vee 0_{\sim}=A$

$\Rightarrow \tau_{i}^{\star}(L)$ is the intuitionistic fuzzy discrete bitopology on $X$.

Theorem. 3.13 :

Let $\left(X, \tau_{1}, \tau_{2}\right)$ be an IFBTS and $L, J$ be two IFIs on $X$. Then for any $A, B \in \operatorname{IFS}(X)$, we have

(i) If $A \leq B \Rightarrow A^{\star}\left(L, \tau_{i}\right) \leq B^{\star}\left(L, \tau_{i}\right), i \in\{1,2\}$.

(ii) If $L \leq J \Rightarrow A^{\star}\left(J, \tau_{i}\right) \leq A^{\star}\left(L, \tau_{i}\right), i \in\{1,2\}$.

(iii) $A^{\star}\left(L, \tau_{i}\right)=\tau_{i}-\operatorname{cl}\left(A^{\star}\right) \leq \tau_{i}-\operatorname{cl}(A), i \in\{1,2\}$.

(iv) $A^{\star \star}\left(L, \tau_{i}\right) \leq A^{\star}\left(L, \tau_{i}\right), i \in\{1,2\}$.

(v) $(A \vee B)^{\star}\left(L, \tau_{i}\right)=A^{\star}\left(L, \tau_{i}\right) \vee B^{\star}\left(L, \tau_{i}\right), i \in\{1,2\}$.

(vi) $(A \wedge B)^{\star}\left(L, \tau_{i}\right)=A^{\star}\left(L, \tau_{i}\right) \wedge B^{\star}\left(L, \tau_{i}\right), i \in\{1,2\}$.

(vii) If $B \in L \Rightarrow(A \vee B)^{\star}\left(L, \tau_{i}\right)=A^{\star}\left(L, \tau_{i}\right), i \in\{1,2\}$.

\section{Proof :}

(i) Let $x_{(\alpha, \beta)} \in A^{\star}\left(L, \tau_{i}\right) \Rightarrow A \wedge U \notin L$, for every $U \in N\left(x_{(\alpha, \beta)}, \tau_{i}\right)$

Since $A \leq B \Rightarrow B \wedge U \notin L$, for every $U \in N\left(x_{(\alpha, \beta)}, \tau_{i}\right) \Rightarrow x_{(\alpha, \beta)} \in B^{\star}\left(L, \tau_{i}\right)$. Therefore $A^{\star}\left(L, \tau_{i}\right) \leq B^{\star}\left(L, \tau_{i}\right)$.

(ii) Let $x_{(\alpha, \beta)} \in A^{\star}\left(J, \tau_{i}\right) \Rightarrow A \wedge U \notin J$, for every $U \in N\left(x_{(\alpha, \beta)}, \tau_{i}\right)$

Since $L \leq J \Rightarrow A \wedge U \notin L$, for every $U \in N\left(x_{(\alpha, \beta)}, \tau_{i}\right) \Rightarrow x_{(\alpha, \beta)} \in A^{\star}\left(L, \tau_{i}\right)$. Thus $A^{\star}\left(J, \tau_{i}\right) \leq A^{\star}\left(L, \tau_{i}\right)$.

(iii) Since $\left\{0_{\sim}\right\} \leq L$, for every IFI on $X \Rightarrow$ from (ii) and (Remark. 3.12:), we get:

$A^{\star}\left(L, \tau_{i}\right) \leq A^{\star}\left(\left\{0_{\sim}\right\}, \tau_{i}\right)=\tau_{i}-\operatorname{cl}(A)$, for every $A \in \operatorname{IFS}(X) \Rightarrow A^{\star}\left(L, \tau_{i}\right) \leq \tau_{i}-\operatorname{cl}(A)$.

Now, we will prove that: $A^{\star}\left(L, \tau_{i}\right)=\tau_{i}-\operatorname{cl}\left(A^{\star}\right)$.

$(\Leftarrow)$ Suppose that $x_{1_{(\alpha, \beta)}} \in \tau_{i}-\operatorname{cl}\left(A^{\star}\right) \Rightarrow A^{\star} \wedge U \neq 0_{\sim}$, for every $U \in N\left(x_{1_{(\alpha, \beta)},}, \tau_{i}\right)$

$\Rightarrow$ there exists $x_{2(\alpha, \beta)} \in A^{\star} \wedge U$, such that $A \wedge V \notin L$, for every $V \in N\left(x_{2(\alpha, \beta)}, \tau_{i}\right)$

Since $U \wedge V \in N\left(x_{2(\alpha, \beta)}, \tau_{i}\right) \Rightarrow A \wedge(U \wedge V) \notin L \Rightarrow A \wedge U \notin L$, for every $U \in N\left(x_{1(\alpha, \beta)}, \tau_{i}\right)$ 
$\Rightarrow x_{1(\alpha, \beta)} \in A^{\star}\left(L, \tau_{i}\right)$. Hence $\tau_{i}-\operatorname{cl}\left(A^{\star}\right) \leq A^{\star}\left(L, \tau_{i}\right) \ldots \ldots(1)$

$(\Rightarrow)$ From (Proposition. 3.10:(v)), we get : $A^{\star}\left(L, \tau_{i}\right) \leq \tau_{i}-\operatorname{cl}\left(A^{\star}\right)$

$\Rightarrow$ From (1) and (2), we have : $A^{\star}\left(L, \tau_{i}\right)=\tau_{i}-\operatorname{cl}\left(A^{\star}\right)$.

(iv) From (iii), we get : $A^{\star \star}\left(L, \tau_{i}\right)=\tau_{i}-\operatorname{cl}\left(A^{\star \star}\right) \leq \tau_{i}-\operatorname{cl}\left(A^{\star}\right)=A^{\star}\left(L, \tau_{i}\right) \Rightarrow A^{\star \star}\left(L, \tau_{i}\right) \leq A^{\star}\left(L, \tau_{i}\right)$.

$(\boldsymbol{v})(\Rightarrow)$ Suppose that $x_{(\alpha, \beta)} \in(A \vee B)^{\star}\left(L, \tau_{i}\right) \Rightarrow(A \vee B) \wedge U \notin L$, for every $U \in N\left(x_{(\alpha, \beta)}, \tau_{i}\right)$

$\Rightarrow(A \wedge U) \vee(B \wedge U) \notin L \Rightarrow A \wedge U \notin L$ and $B \wedge U \in L$ or $A \wedge U \in L$ and $B \wedge U \notin L$

$\Rightarrow A \wedge U \notin L \quad$ or $B \wedge U \notin L$, for every $U \in N\left(x_{(\alpha, \beta)}, \tau_{i}\right)$

$\Rightarrow x_{(\alpha, \beta)} \in A^{\star}\left(L, \tau_{i}\right)$ or $x_{(\alpha, \beta)} \in B^{\star}\left(L, \tau_{i}\right) \Rightarrow x_{(\alpha, \beta)} \in A^{\star}\left(L, \tau_{i}\right) \vee B^{\star}\left(L, \tau_{i}\right) \Rightarrow(A \vee B)^{\star}\left(L, \tau_{i}\right) \leq A^{\star}\left(L, \tau_{i}\right) \vee B^{\star}\left(L, \tau_{i}\right) \ldots \ldots(1)$

$(\Leftrightarrow)$ Since $A \leq A \vee B$ and $B \leq A \vee B \Rightarrow$ From $(i)$, we get $: A^{\star}\left(L, \tau_{i}\right) \leq(A \vee B)^{\star}\left(L, \tau_{i}\right)$ and $B^{\star}\left(L, \tau_{i}\right) \leq(A \vee B)^{\star}\left(L, \tau_{i}\right)$ $\Rightarrow A^{\star}\left(L, \tau_{i}\right) \vee B^{\star}\left(L, \tau_{i}\right) \leq(A \vee B)^{\star}\left(L, \tau_{i}\right) \ldots \ldots(2)$

$\Rightarrow$ From (1) and (2), we get : $(A \vee B)^{\star}\left(L, \tau_{i}\right)=A^{\star}\left(L, \tau_{i}\right) \vee B^{\star}\left(L, \tau_{i}\right)$.

(vi) $(\Rightarrow)$ Since $A \wedge B \leq A$ and $A \wedge B \leq B \Rightarrow$ from $(i)$, we get $:(A \wedge B)^{\star}\left(L, \tau_{i}\right) \leq A^{\star}\left(L, \tau_{i}\right)$ and $(A \wedge B)^{\star}\left(L, \tau_{i}\right) \leq B^{\star}\left(L, \tau_{i}\right)$ $\Rightarrow(A \wedge B)^{\star}\left(L, \tau_{i}\right) \leq A^{\star}\left(L, \tau_{i}\right) \wedge B^{\star}\left(L, \tau_{i}\right)$

$(\Leftarrow)$ Suppose that $x_{(\alpha, \beta)} \in A^{\star}\left(L, \tau_{i}\right) \wedge B^{\star}\left(L, \tau_{i}\right) \Rightarrow x_{(\alpha, \beta)} \in A^{\star}\left(L, \tau_{i}\right)$ and $x_{(\alpha, \beta)} \in B^{\star}\left(L, \tau_{i}\right)$

$\Rightarrow A \wedge U \notin L \quad$ and $B \wedge U \notin L$, for every $U \in N\left(x_{(\alpha, \beta)}, \tau_{i}\right) \Rightarrow(A \wedge U) \wedge(B \wedge U) \notin L$, for every $U \in N\left(x_{(\alpha, \beta)}, \tau_{i}\right)$

$\Rightarrow(A \wedge B) \wedge U \notin L$, for every $U \in N\left(x_{(\alpha, \beta)}, \tau_{i}\right) \Rightarrow x_{(\alpha, \beta)} \in(A \wedge B)^{\star}\left(L, \tau_{i}\right)$

$\Rightarrow A^{\star}\left(L, \tau_{i}\right) \wedge B^{\star}\left(L, \tau_{i}\right) \leq(A \wedge B)^{\star}\left(L, \tau_{i}\right)$.....

$\Rightarrow$ From (1) and (2), we get $:(A \wedge B)^{\star}\left(L, \tau_{i}\right)=A^{\star}\left(L, \tau_{i}\right) \wedge B^{\star}\left(L, \tau_{i}\right)$

(vii) $(\Rightarrow)$ Suppose that $x_{(\alpha, \beta)} \in(A \vee B)^{\star}\left(L, \tau_{i}\right) \Rightarrow$ from $(v)$, we have $: x_{(\alpha, \beta)} \in A^{\star}\left(L, \tau_{i}\right) \vee B^{\star}\left(L, \tau_{i}\right)$

$\Rightarrow x_{(\alpha, \beta)} \in A^{\star}\left(L, \tau_{i}\right)$ or $x_{(\alpha, \beta)} \in B^{\star}\left(L, \tau_{i}\right)$

If $x_{(\alpha, \beta)} \in A^{\star}\left(L, \tau_{i}\right) \Rightarrow(A \vee B)^{\star}\left(L, \tau_{i}\right) \leq A^{\star}\left(L, \tau_{i}\right) \ldots \ldots\left(*_{1}\right)$

If $x_{(\alpha, \beta)} \in B^{\star}\left(L, \tau_{i}\right) \Rightarrow B \wedge U \notin L$, for every $U \in N\left(x_{(\alpha, \beta)}, \tau_{i}\right)$

Since $B \in L$, and $B \wedge U \notin L \Rightarrow U \notin L \Rightarrow A \wedge U \notin L$, for every $U \in N\left(x_{(\alpha, \beta)}, \tau_{i}\right) \Rightarrow x_{(\alpha, \beta)} \in A^{\star}\left(L, \tau_{i}\right)$ $\Rightarrow(A \vee B)^{\star}\left(L, \tau_{i}\right) \leq A^{\star}\left(L, \tau_{i}\right) \ldots \ldots\left(*_{2}\right)$

$(\Leftarrow)$ Since $A \leq A \vee B \Rightarrow$ from $(i)$, we have : $A^{\star}\left(L, \tau_{i}\right) \leq(A \vee B)^{\star}\left(L, \tau_{i}\right) \ldots \ldots(* *)$

$\Rightarrow$ From $\left(*_{1}\right),\left(*_{2}\right)$ and $(* *)$, we get $:(A \vee B)^{\star}\left(L, \tau_{i}\right)=A^{\star}\left(L, \tau_{i}\right)$.

\section{Theorem. 3.14 :}

Let $\left(X, \tau_{1}, \tau_{2}, L\right)$ be an IFIBTS, $A, B \in \operatorname{IFS}(X)$ and $\delta=\tau_{1} \vee \tau_{2}$ be an intuitionistic fuzzy topology generated by $\tau_{1}, \tau_{2}$. Then we have:

(i) $A^{\star}(L, \delta) \leq A^{\star}\left(L, \tau_{i}\right), i \in\{1,2\}$.

(ii) If $A \leq B \Rightarrow A^{\star}(L, \delta) \leq B^{\star}\left(L, \tau_{i}\right), i \in\{1,2\}$.

(iii) $A^{\star}(L, \delta) \leq \delta-\operatorname{cl}(A) \leq \tau_{i}-\operatorname{cl}(A), i \in\{1,2\}$.

(iv) $A^{\star \star}(L, \delta) \leq A^{\star}\left(L, \tau_{i}\right), i \in\{1,2\}$.

\section{Proof :}

(i) Let $x_{(\alpha, \beta)} \notin A^{\star}\left(L, \tau_{i}\right) \Rightarrow$ there exists $U \in N\left(x_{(\alpha, \beta)}, \tau_{i}\right)$ such that $A \wedge U \in L$

Since $\tau_{i} \leq \delta=\tau_{1} \vee \tau_{2} \Rightarrow N\left(x_{(\alpha, \beta)}, \tau_{i}\right) \leq N\left(x_{(\alpha, \beta)}, \delta\right)$

$\Rightarrow U \in N\left(x_{(\alpha, \beta)}, \delta\right)$ and $A \wedge U \in L \Rightarrow x_{(\alpha, \beta)} \notin A^{\star}(L, \delta) \Rightarrow A^{\star}(L, \delta) \leq A^{\star}\left(L, \tau_{i}\right)$.

(ii) Let $x_{(\alpha, \beta)} \in A^{\star}(L, \delta) \Rightarrow A \wedge U \notin L$, for every $U \in N\left(x_{(\alpha, \beta)}, \delta\right)$

Since $A \leq B \Rightarrow B \wedge U \notin L$, for every $U \in N\left(x_{(\alpha, \beta)}, \delta\right) \Rightarrow x_{(\alpha, \beta)} \in B^{\star}(L, \delta) \Rightarrow A^{\star}(L, \delta) \leq B^{\star}(L, \delta)$ 
$\Rightarrow$ from $(i)$, we get : $B^{\star}(L, \delta) \leq B^{\star}\left(L, \tau_{i}\right), i \in\{1,2\}$. Therefore $A^{\star}(L, \delta) \leq B^{\star}\left(L, \tau_{i}\right), i \in\{1,2\}$.

(iii) From ( Theorem. 3.13:(iii)), we have : $A^{\star}(L, \delta) \leq \delta-\operatorname{cl}(A)$

Since $\tau_{i} \leq \delta=\tau_{1} \vee \tau_{2} \Rightarrow \delta-\operatorname{cl}(A) \leq \tau_{i}-\operatorname{cl}(A)$. Hence $A^{\star}(L, \delta) \leq \delta-\operatorname{cl}(A) \leq \tau_{i}-\operatorname{cl}(A)$

(iv) From ( Theorem. 3.13:(iv)), we get : $A^{\star \star}(L, \delta) \leq A^{\star}(L, \delta)$

$\Rightarrow$ from $(i)$, we have : $A^{\star}(L, \delta) \leq A^{\star}\left(L, \tau_{i}\right), i \in\{1,2\}$. Thus $A^{\star \star}(L, \delta) \leq A^{\star}\left(L, \tau_{i}\right), i \in\{1,2\}$.

\section{Lemma. 3.15 :}

Let $\left(X, \tau_{1}, \tau_{2}, L\right)$ be an IFIBTS, $A \in \operatorname{IFS}(X)$. Then we have : $\tau_{i}-c l^{\star}(A) \leq \tau_{i}-\operatorname{cl}(A), i \in\{1,2\}$.

\section{Proof :}

From (Theorem.3.13: (iii) ), we get : $A^{\star}\left(L, \tau_{i}\right) \leq \tau_{i}-\operatorname{cl}(A) \Rightarrow A \vee A^{\star}\left(L, \tau_{i}\right) \leq A \vee \tau_{i}-\operatorname{cl}(A)$

Since $A \leq \tau_{i}-\operatorname{cl}(A) \Rightarrow A \vee \tau_{i}-\operatorname{cl}(A)=\tau_{i}-\operatorname{cl}(A) \Rightarrow A \vee A^{\star}\left(L, \tau_{i}\right) \leq \tau_{i}-\operatorname{cl}(A)$

Since $\tau_{i}-c l^{\star}(A)=A \vee A^{\star}\left(L, \tau_{i}\right) \Rightarrow \tau_{i}-c l^{\star}(A) \leq \tau_{i}-c l(A)$.

\section{Theorem. 3.16 :}

Let $\left(X, \tau_{1}, \tau_{2}, L\right)$ be an IFIBTS, $A \in \operatorname{IFS}(X)$ and $\tau_{1} \leq \tau_{2}$. Then :

(i) $A^{\star}\left(L, \tau_{2}\right) \leq A^{\star}\left(L, \tau_{1}\right)$.

(ii) $\tau_{1}{ }^{\star}(L) \leq \tau_{2}{ }^{\star}(L)$.

(iii) $A^{\star}\left(L, \tau_{1}{ }^{\star}(L)\right) \leq A^{\star}\left(L, \tau_{2}{ }^{\star}(L)\right)$.

\section{Proof :}

(i) Suppose that $x_{(\alpha, \beta)} \notin A^{\star}\left(L, \tau_{1}\right) \Rightarrow$ there exists $U \in N\left(x_{(\alpha, \beta)}, \tau_{1}\right)$ such that $A \wedge U \in L$.

Since $\tau_{1} \leq \tau_{2} \Rightarrow U \in N\left(x_{(\alpha, \beta)}, \tau_{2}\right)$

Now, $U \in N\left(x_{(\alpha, \beta)}, \tau_{2}\right)$ and $A \wedge U \in L \Rightarrow x_{(\alpha, \beta)} \notin A^{\star}\left(L, \tau_{2}\right)$. Therefore $A^{\star}\left(L, \tau_{2}\right) \leq A^{\star}\left(L, \tau_{1}\right)$.

(ii) Let $A \in \tau_{1}^{\star}(L) \Rightarrow$ from (Definition. 3.11: ), we get : $\tau_{1}-c l^{\star}\left(A^{c}\right)=A^{c} \Rightarrow A^{c} \vee\left(A^{c}\right)^{\star}\left(L, \tau_{1}\right)=A^{c}$

Now , $\tau_{2}-c l^{\star}\left(A^{c}\right)=A^{c} \vee\left(A^{c}\right)^{\star}\left(L, \tau_{2}\right)$

$\Rightarrow$ From $(i)$, we have : $A^{c} \vee\left(A^{c}\right)^{\star}\left(L, \tau_{2}\right) \leq A^{c} \vee\left(A^{c}\right)^{\star}\left(L, \tau_{1}\right)=A^{c} \Rightarrow \tau_{2}-c l^{\star}\left(A^{c}\right) \leq A^{c}$

Since $A^{c} \leq \tau_{2}-c l^{\star}\left(A^{c}\right) \ldots \ldots(2)$

$\Rightarrow$ From (1) and (2), we get : $\tau_{2}-c l^{\star}\left(A^{c}\right)=A^{c} \Rightarrow A \in \tau_{2}{ }^{\star}(L)$. Thus $\tau_{1}{ }^{\star}(L) \leq \tau_{2}{ }^{\star}(L)$.

(iii)Suppose that $x_{(\alpha, \beta)} \in A^{\star}\left(L, \tau_{1}{ }^{\star}(L)\right) \Rightarrow A \wedge U \notin L$, for every $U \in N\left(x_{(\alpha, \beta)}, \tau_{1}{ }^{\star}(L)\right)$

$\Rightarrow$ From $(i i)$, we have : $U \in N\left(x_{(\alpha, \beta)}, \tau_{2}{ }^{\star}(L)\right) \Rightarrow A \wedge U \notin L$, for every $U \in N\left(x_{(\alpha, \beta)}, \tau_{2}{ }^{\star}(L)\right) \Rightarrow x_{(\alpha, \beta)} \in A^{\star}\left(L, \tau_{2}{ }^{\star}(L)\right)$ $\Rightarrow A^{\star}\left(L, \tau_{1}{ }^{\star}(L)\right) \leq A^{\star}\left(L, \tau_{2}{ }^{\star}(L)\right)$.

\section{Theorem. 3.17:}

Let $L_{1}$ and $L_{2}$ be two IFIs on $\operatorname{IFBTS}\left(X, \tau_{1}, \tau_{2}\right)$. Then we have:

(i) If $L_{1} \leq L_{2} \Rightarrow \tau_{i}^{\star}\left(L_{1}\right) \leq \tau_{i}^{\star}\left(L_{2}\right), i \in\{1,2\}$.

(ii) $A^{\star}\left(L_{1} \wedge L_{2}, \tau_{i}\right)=A^{\star}\left(L_{1}, \tau_{i}\right) \vee A^{\star}\left(L_{2}, \tau_{i}\right), i \in\{1,2\}$.

(iii) $A^{\star}\left(L_{1} \vee L_{2}, \tau_{i}\right)=A^{\star}\left(L_{1}, \tau_{i}\right) \wedge A^{\star}\left(L_{2}, \tau_{i}\right), i \in\{1,2\}$.

(iv) $\tau_{i}^{\star}\left(L_{1} \wedge L_{2}\right)=\tau_{i}^{\star}\left(L_{1}\right) \wedge \tau_{i}^{\star}\left(L_{2}\right), i \in\{1,2\}$.

(v) $\tau_{i}^{\star}\left(L_{1} \vee L_{2}\right)=\tau_{i}^{\star}\left(L_{1}\right) \vee \tau_{i}^{\star}\left(L_{2}\right), i \in\{1,2\}$. 


\section{Proof :}

(i) Suppose that $A \in \tau_{i}{ }^{\star}\left(L_{1}\right) \Rightarrow$ from (Definition. 3.11:), we have : $\tau_{i}-c l^{\star}\left(A^{c}\right)=A^{c}$

$\Rightarrow A^{c} \vee\left(A^{c}\right)^{\star}\left(L_{1}, \tau_{i}\right)=A^{c} \Rightarrow\left(A^{c}\right)^{\star}\left(L_{1}, \tau_{i}\right) \leq A^{c}$

Since $L_{1} \leq L_{2} \Rightarrow$ from (Theorem.3.13: (ii)), we get : $\left(A^{c}\right)^{\star}\left(L_{2}, \tau_{i}\right) \leq\left(A^{c}\right)^{\star}\left(L_{1}, \tau_{i}\right)$

$\Rightarrow\left(A^{c}\right)^{\star}\left(L_{2}, \tau_{i}\right) \leq A^{c} \Rightarrow A^{c} \vee\left(A^{c}\right)^{\star}\left(L_{2}, \tau_{i}\right)=A^{c} \Rightarrow A \in \tau_{i}{ }^{\star}\left(L_{2}\right)$. Thus $\tau_{i}^{\star}\left(L_{1}\right) \leq \tau_{i}^{\star}\left(L_{2}\right)$.

$(i i)(\Rightarrow)$ Let $x_{(\alpha, \beta)} \in A^{\star}\left(L_{1} \wedge L_{2}, \tau_{i}\right) \Rightarrow A \wedge U \notin\left(L_{1} \wedge L_{2}\right)$, for every $U \in N\left(x_{(\alpha, \beta)}, \tau_{i}\right)$

$\Rightarrow A \wedge U \notin L_{1} \quad$ or $A \wedge U \notin L_{2}$, for every $U \in N\left(x_{(\alpha, \beta)}, \tau_{i}\right)$

$\Rightarrow x_{(\alpha, \beta)} \in A^{\star}\left(L_{1}, \tau_{i}\right)$ or $x_{(\alpha, \beta)} \in A^{\star}\left(L_{2}, \tau_{i}\right) \Rightarrow x_{(\alpha, \beta)} \in A^{\star}\left(L_{1}, \tau_{i}\right) \vee A^{\star}\left(L_{2}, \tau_{i}\right)$

$A^{\star}\left(L_{1} \wedge L_{2}, \tau_{i}\right) \leq A^{\star}\left(L_{1}, \tau_{i}\right) \vee A^{\star}\left(L_{2}, \tau_{i}\right)$

$(\Leftarrow)$ Since $L_{1} \wedge L_{2} \leq L_{1}$ and $L_{1} \wedge L_{2} \leq L_{2} \Rightarrow$ From(Theorem. 3.13: (ii)), we have:

$A^{\star}\left(L_{1}, \tau_{i}\right) \leq A^{\star}\left(L_{1} \wedge L_{2}, \tau_{i}\right)$ and $A^{\star}\left(L_{2}, \tau_{i}\right) \leq A^{\star}\left(L_{1} \wedge L_{2}, \tau_{i}\right) \Rightarrow A^{\star}\left(L_{1}, \tau_{i}\right) \vee A^{\star}\left(L_{2}, \tau_{i}\right) \leq A^{\star}\left(L_{1} \wedge L_{2}, \tau_{i}\right)$.

$\Rightarrow$ From (1) and (2) $\Rightarrow A^{\star}\left(L_{1} \wedge L_{2}, \tau_{i}\right)=A^{\star}\left(L_{1}, \tau_{i}\right) \vee A^{\star}\left(L_{2}, \tau_{i}\right)$.

(iii) $\Leftrightarrow)$ Since $L_{1} \leq L_{1} \vee L_{2}$ and $L_{2} \leq L_{1} \vee L_{2}$

$\Rightarrow$ From (Theorem. 3.13: (ii)), we have : $A^{\star}\left(L_{1} \vee L_{2}, \tau_{i}\right) \leq A^{\star}\left(L_{1}, \tau_{i}\right)$ and $A^{\star}\left(L_{1} \vee L_{2}, \tau_{i}\right) \leq A^{\star}\left(L_{2}, \tau_{i}\right)$.

$\Rightarrow A^{\star}\left(L_{1} \vee L_{2}, \tau_{i}\right) \leq A^{\star}\left(L_{1}, \tau_{i}\right) \wedge A^{\star}\left(L_{2}, \tau_{i}\right)$......

$(\Leftarrow)$ Suppose that $x_{(\alpha, \beta)} \in A^{\star}\left(L_{1}, \tau_{i}\right) \wedge A^{\star}\left(L_{2}, \tau_{i}\right) \Rightarrow x_{(\alpha, \beta)} \in A^{\star}\left(L_{1}, \tau_{i}\right)$ and $x_{(\alpha, \beta)} \in A^{\star}\left(L_{2}, \tau_{i}\right)$

$\Rightarrow A \wedge U \notin L_{1}$ and $A \wedge U \notin L_{2}$, for every $U \in N\left(x_{(\alpha, \beta)}, \tau_{i}\right)$

$\Rightarrow A \wedge U \notin\left(L_{1} \vee L_{2}\right)$, for every $U \in N\left(x_{(\alpha, \beta)}, \tau_{i}\right) \Rightarrow x_{(\alpha, \beta)} \in A^{\star}\left(L_{1} \vee L_{2}, \tau_{i}\right)$

$\Rightarrow A^{\star}\left(L_{1}, \tau_{i}\right) \wedge A^{\star}\left(L_{2}, \tau_{i}\right) \leq A^{\star}\left(L_{1} \vee L_{2}, \tau_{i}\right) \ldots \ldots(2)$

$\Rightarrow$ From (1) and (2) $\Rightarrow A^{\star}\left(L_{1} \vee L_{2}, \tau_{i}\right)=A^{\star}\left(L_{1}, \tau_{i}\right) \wedge A^{\star}\left(L_{2}, \tau_{i}\right)$.

(iv) Since $L_{1} \wedge L_{2} \leq L_{1}$ and $L_{1} \wedge L_{2} \leq L_{2} \Rightarrow$ from $(i)$, we get : $\tau_{i}^{\star}\left(L_{1} \wedge L_{2}\right) \leq \tau_{i}^{\star}\left(L_{1}\right)$ and $\tau_{i}^{\star}\left(L_{1} \wedge L_{2}\right) \leq \tau_{i}^{\star}\left(L_{2}\right)$ $\Rightarrow \tau_{i}^{\star}\left(L_{1} \wedge L_{2}\right) \leq \tau_{i}^{\star}\left(L_{1}\right) \wedge \tau_{i}^{\star}\left(L_{2}\right)$.....

$(\Leftarrow)$ Suppose that $A \in\left[\tau_{i}^{\star}\left(L_{1}\right) \wedge \tau_{i}^{\star}\left(L_{2}\right)\right] \Rightarrow A \in \tau_{i}^{\star}{ }^{\star}\left(L_{1}\right)$ and $A \in \tau_{i}^{\star}\left(L_{2}\right)$

$\Rightarrow$ From (Definition. 3.11: ), we get : $A^{c} \vee\left(A^{c}\right)^{\star}\left(L_{1}, \tau_{i}\right)=A^{c}$ and $A^{c} \vee\left(A^{c}\right)^{\star}\left(L_{2}, \tau_{i}\right)=A^{c}$

$\Rightarrow\left[A^{c} \vee\left(A^{c}\right)^{\star}\left(L_{1}, \tau_{i}\right)\right] \vee\left[A^{c} \vee\left(A^{c}\right)^{\star}\left(L_{2}, \tau_{i}\right)\right]=A^{c}$

$\Rightarrow A^{c} \vee\left[\left(A^{c}\right)^{\star}\left(L_{1}, \tau_{i}\right) \vee\left(A^{c}\right)^{\star}\left(L_{2}, \tau_{i}\right)\right]=A^{c} \Rightarrow$ from $(i i)$, we get : $A^{c} \vee\left(A^{c}\right)^{\star}\left(L_{1} \wedge L_{2}, \tau_{i}\right)=A^{c} \Rightarrow A \in \tau_{i}^{\star}\left(L_{1} \wedge L_{2}\right)$

$\Rightarrow$ From (1) and (2), we get : $\tau_{i}^{\star}\left(L_{1} \wedge L_{2}\right)=\tau_{i}^{\star}\left(L_{1}\right) \wedge \tau_{i}^{\star}\left(L_{2}\right)$.

$(\boldsymbol{v})(\Rightarrow)$ Suppose that $A \in \tau_{i}^{\star}\left(L_{1} \vee L_{2}\right) \Rightarrow$ From (Definition.3.11: ), we get : $A^{c} \vee\left(A^{c}\right)^{\star}\left(L_{1} \vee L_{2}, \tau_{i}\right)=A^{c}$

$\Rightarrow$ From (iii), we have : $A^{c} \vee\left[\left(A^{c}\right)^{\star}\left(L_{1}, \tau_{i}\right) \wedge\left(A^{c}\right)^{\star}\left(L_{2}, \tau_{i}\right)\right]=A^{c}$

$\Rightarrow\left[A^{c} \vee\left(A^{c}\right)^{\star}\left(L_{1}, \tau_{i}\right)\right] \wedge\left[A^{c} \vee\left(A^{c}\right)^{\star}\left(L_{2}, \tau_{i}\right)\right]=A^{c} \Rightarrow A^{c} \vee\left(A^{c}\right)^{\star}\left(L_{1}, \tau_{i}\right)=A^{c}$ and $A^{c} \vee\left(A^{c}\right)^{\star}\left(L_{2}, \tau_{i}\right)=A^{c}$

$\Rightarrow$ From (Definition. 3.11:), we get : $A \in \tau_{i}^{\star}\left(L_{1}\right)$ and $A \in \tau_{i}^{\star}\left(L_{2}\right)$

$\Rightarrow A \in\left[\tau_{i}^{\star}\left(L_{1}\right) \vee \tau_{i}^{\star}\left(L_{2}\right)\right] \Rightarrow \tau_{i}^{\star}\left(L_{1} \vee L_{2}\right) \leq \tau_{i}^{\star}\left(L_{1}\right) \vee \tau_{i}^{\star}\left(L_{2}\right)$

$(\Leftarrow)$ Since $L_{1} \leq L_{1} \vee L_{2}$ and $L_{2} \leq L_{1} \vee L_{2} \Rightarrow$ from $(i)$, we get : $\tau_{i}^{\star}\left(L_{1}\right) \leq \tau_{i}^{\star}\left(L_{1} \vee L_{2}\right)$ and $\tau_{i}^{\star}\left(L_{2}\right) \leq \tau_{i}^{\star}\left(L_{1} \vee L_{2}\right)$ $\Rightarrow \tau_{i}^{\star}\left(L_{1}\right) \vee \tau_{i}^{\star}\left(L_{2}\right) \leq \tau_{i}^{\star}\left(L_{1} \vee L_{2}\right) \ldots \ldots(2) \Rightarrow$ from (1) and (2), we have : $\tau_{i}^{\star}\left(L_{1} \vee L_{2}\right)=\tau_{i}^{\star}\left(L_{1}\right) \vee \tau_{i}^{\star}\left(L_{2}\right)$.

\section{Theorem. 3.18 :}

(i) $A^{\star}\left(L_{1} \vee L_{2}, \tau_{i}\right)=A^{\star}\left(L_{1}, \tau_{i}^{\star}\left(L_{1}\right)\right) \wedge A^{\star}\left(L_{2}, \tau_{i}^{\star}\left(L_{2}\right)\right), i \in\{1,2\}$.

(ii) $A^{\star}\left(L_{1} \wedge L_{2}, \tau_{i}\right)=A^{\star}\left(L_{1}, \tau_{i}^{\star}\left(L_{1}\right)\right) \vee A^{\star}\left(L_{2}, \tau_{i}^{\star}\left(L_{2}\right)\right), i \in\{1,2\}$.

(iii) $\tau_{i}^{\star}\left(L_{1} \vee L_{2}\right)=\tau_{i}^{\star \star}\left(L_{1}\right) \wedge \tau_{i}^{\star \star}\left(L_{2}\right), i \in\{1,2\}$.

(iv) $\tau_{i}^{\star}\left(L_{1} \wedge L_{2}\right)=\tau_{i}^{\star \star}\left(L_{1}\right) \vee \tau_{i}^{\star \star}\left(L_{2}\right), i \in\{1,2\}$. 


\section{Proof :}

(i) $(\Rightarrow)$ Let $x_{(\alpha, \beta)} \notin\left[A^{\star}\left(L_{1}, \tau_{i}^{\star}\left(L_{1}\right)\right) \wedge A^{\star}\left(L_{2}, \tau_{i}^{\star}\left(L_{2}\right)\right)\right] \Rightarrow x_{(\alpha, \beta)} \notin A^{\star}\left(L_{1}, \tau_{i}{ }^{\star}\left(L_{1}\right)\right)$ or $x_{(\alpha, \beta)} \notin A^{\star}\left(L_{2}, \tau_{i}{ }^{\star}\left(L_{2}\right)\right)$

$\Rightarrow A \wedge U_{1} \in L_{1}$, for some $U_{1} \in N\left(x_{(\alpha, \beta)}, \tau_{i}^{*}\left(L_{1}\right)\right)$ or $A \wedge U_{2} \in L_{2}$, for some $U_{2} \in N\left(x_{(\alpha, \beta)}, \tau_{i}{ }^{\star}\left(L_{2}\right)\right)$

$\Rightarrow\left(A \wedge U_{1}\right) \vee\left(A \wedge U_{2}\right) \in\left(L_{1} \vee L_{2}\right) \Rightarrow A \wedge\left(U_{1} \vee U_{2}\right) \in\left(L_{1} \vee L_{2}\right)$

Since $U_{1} \in N\left(x_{(\alpha, \beta)}, \tau_{i}^{\star}\left(L_{1}\right)\right)$ and $\tau_{i} \leq \tau_{i}^{\star}{ }^{\star}\left(L_{1}\right) \Rightarrow U_{1} \in N\left(x_{(\alpha, \beta)}, \tau_{i}\right)$

Since $U_{2} \in N\left(x_{(\alpha, \beta)}, \tau_{i}^{\star}\left(L_{2}\right)\right)$ and $\tau_{i} \leq \tau_{i}^{\star}{ }^{\star}\left(L_{2}\right) \Rightarrow U_{2} \in N\left(x_{(\alpha, \beta)}, \tau_{i}\right)$

$\Rightarrow\left(U_{1} \vee U_{2}\right) \in N\left(x_{(\alpha, \beta)}, \tau_{i}\right) \Rightarrow A \wedge\left(U_{1} \vee U_{2}\right) \in\left(L_{1} \vee L_{2}\right)$, for some $\left(U_{1} \vee U_{2}\right) \in N\left(x_{(\alpha, \beta)}, \tau_{i}\right)$

$\Rightarrow x_{(\alpha, \beta)} \notin A^{\star}\left(L_{1} \vee L_{2}, \tau_{i}\right) \Rightarrow A^{\star}\left(L_{1} \vee L_{2}, \tau_{i}\right) \leq A^{\star}\left(L_{1}, \tau_{i}^{\star}\left(L_{1}\right)\right) \wedge A^{\star}\left(L_{2}, \tau_{i}^{\star}\left(L_{2}\right)\right) \ldots$

$(\Leftarrow)$ From (Definition.3.11:), we have : $\tau_{i} \leq \tau_{i}{ }^{\star}\left(L_{1}\right)$ and $\tau_{i} \leq \tau_{i}{ }^{\star}\left(L_{2}\right)$

$\Rightarrow$ From (Theorem.3.16:(i)), we get : $A^{\star}\left(L_{1}, \tau_{i}^{\star}\left(L_{1}\right)\right) \leq A^{\star}\left(L_{1}, \tau_{i}\right)$ and $A^{\star}\left(L_{2}, \tau_{i}^{\star}\left(L_{2}\right)\right) \leq A^{\star}\left(L_{2}, \tau_{i}\right)$

$\Rightarrow A^{\star}\left(L_{1}, \tau_{i}^{\star}\left(L_{1}\right)\right) \wedge A^{\star}\left(L_{2}, \tau_{i}^{\star}\left(L_{2}\right)\right) \leq A^{\star}\left(L_{1}, \tau_{i}\right) \wedge A^{\star}\left(L_{2}, \tau_{i}\right)$

$\Rightarrow$ From (Theorem.3.17: (iii) ), we have : $A^{\star}\left(L_{1}, \tau_{i}^{\star}\left(L_{1}\right)\right) \wedge A^{\star}\left(L_{2}, \tau_{i}^{\star}\left(L_{2}\right)\right) \leq A^{\star}\left(L_{1} \vee L_{2}, \tau_{i}\right) \ldots$

$\Rightarrow$ From (1) and (2), we get : $A^{\star}\left(L_{1} \vee L_{2}, \tau_{i}\right)=A^{\star}\left(L_{1}, \tau_{i}^{\star}\left(L_{1}\right)\right) \wedge A^{\star}\left(L_{2}, \tau_{i}^{\star}\left(L_{2}\right)\right)$.

(ii) Similarly as $(i)$.

(iii) By using (i), we get : $\tau_{i}{ }^{\star}\left(L_{1} \vee L_{2}\right)=\tau_{i}^{\star \star}\left(L_{1}\right) \wedge \tau_{i}^{\star \star}\left(L_{2}\right)$.

(iv) By using (ii), we get : $\tau_{i}^{\star}\left(L_{1} \wedge L_{2}\right)=\tau_{i}^{\star \star}\left(L_{1}\right) \vee \tau_{i}^{\star \star}\left(L_{2}\right)$.

\section{Corollary. 3.19 :}

Let $\left(X, \tau_{1}, \tau_{2}, L\right)$ be an IFIBTS. Then we have :

(i) $A^{\star}\left(L, \tau_{i}\right)=A^{\star}\left(L, \tau_{i}^{*}(L)\right), i \in\{1,2\}$.

(ii) $\tau_{i}^{\star}(L)=\tau_{i}^{\star \star}(L), i \in\{1,2\}$.

\section{Proof :}

By taking $L_{1}=L_{2}=L$ in above Theorem, we have the required result.

\section{ACKNOWLEDGEMENT :}

Authors would like to thank the referee for his comments and suggestions to have the manuscript done perfectly and for assistance in responding to concerns and questions regarding my paper.

\section{REFERENCES :}

[1] K. Atanassov ; Intuitionistic Fuzzy Sets, Fuzzy Sets and Systems . 20, 87-96 .(1986) .

[2] M.E. Abd El-Monsef, A. Kozae, A. A. Salama and H.M. Elagmy ; Fuzzy Bitopological Ideals Spaces, Journal of Computer Engineering, ISSN. 2278-0661, ISBN 2278-8727, Vol . 6, ISSUE 4, 1-5 . (2012) .

[3] D. Coker and M. Demirci ; On Intuitionistic Fuzzy Points . NIFSI . 2 , 79-84 . (1995).

[4] D. Coker ; An Introduction to Intuitionistic Fuzzy Topological Spaces, Fuzzy Sets and Systems . 88, 81-89. (1997) .

[5] A. Kandil , A. A. Nouh and S. A. El-Sheikh ; On Fuzzy Bitopological Spaces, Fuzzy Sets and Systems . 74 , 353-363 . (1995) .

[6] A.A. Salama and S.A. Alblowi ; Intuitionistic Fuzzy Ideals Topological Spaaces, ISSN . 0973-533X . Vol.7, No . 1. 51-60 . (2012)

[7] L. A . Zadeh ; Fuzzy Sets Information and Control . 8 , 338- 353. (1965). 